\title{
Front Matter: Volume 11271
}

, "Front Matter: Volume 11271," Proc. SPIE 11271, Laser 3D Manufacturing VII, 1127101 (25 March 2020); doi: 10.1117/12.2569744

SPIE. Event: SPIE LASE, 2020, San Francisco, California, United States 


\section{PROCEEDINGS OF SPIE}

\section{Laser 3D Manufacturing VII}

Bo Gu

Hongqiang Chen

Henry Helvajian

Editors

4-6 February 2020

San Francisco, California, United States

Sponsored and Published by

SPIE 
The papers in this volume were part of the technical conference cited on the cover and title page. Papers were selected and subject to review by the editors and conference program committee. Some conference presentations may not be available for publication. Additional papers and presentation recordings may be available online in the SPIE Digital Library at SPIEDigitallibrary.org.

The papers reflect the work and thoughts of the authors and are published herein as submitted. The publisher is not responsible for the validity of the information or for any outcomes resulting from reliance thereon.

Please use the following format to cite material from these proceedings:

Author(s), 'Title of Paper," in Laser 3D Manufacturing VII, edited by Bo Gu, Hongqiang Chen, Henry Helvajian, Proceedings of SPIE Vol. 11271 (SPIE, Bellingham, WA, 2020) Seven-digit Article CID Number.

ISSN: 0277-786X

ISSN: 1996-756X (electronic)

ISBN: 9781510633056

ISBN: 9781510633063 (electronic)

Published by

SPIE

P.O. Box 10, Bellingham, Washington 98227-0010 USA

Telephone +1 3606763290 (Pacific Time) · Fax + 13606471445

SPIE.org

Copyright @ 2020, Society of Photo-Optical Instrumentation Engineers.

Copying of material in this book for internal or personal use, or for the internal or personal use of specific clients, beyond the fair use provisions granted by the U.S. Copyright Law is authorized by SPIE subject to payment of copying fees. The Transactional Reporting Service base fee for this volume is $\$ 21.00$ per article (or portion thereof), which should be paid directly to the Copyright Clearance Center (CCC), 222 Rosewood Drive, Danvers, MA 01923. Payment may also be made electronically through CCC Online at copyright.com. Other copying for republication, resale, advertising or promotion, or any form of systematic or multiple reproduction of any material in this book is prohibited except with permission in writing from the publisher. The CCC fee code is 0277$786 \times / 20 / \$ 21.00$.

Printed in the United States of America by Curran Associates, Inc., under license from SPIE.

Publication of record for individual papers is online in the SPIE Digital Library.

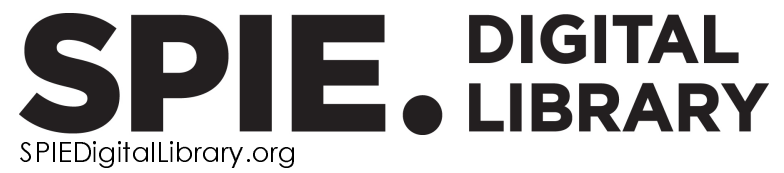

Paper Numbering: Proceedings of SPIE follow an e-First publication model. A unique citation identifier (CID) number is assigned to each article at the time of publication. Utilization of CIDs allows articles to be fully citable as soon as they are published online, and connects the same identifier to all online and print versions of the publication. SPIE uses a seven-digit CID article numbering system structured as follows:

- The first five digits correspond to the SPIE volume number.

- The last two digits indicate publication order within the volume using a Base 36 numbering system employing both numerals and letters. These two-number sets start with $00,01,02,03,04$, 05, 06, 07, 08, 09, OA, OB ... 0Z, followed by 10-1Z, 20-2Z, etc. The CID Number appears on each page of the manuscript. 


\title{
Contents
}

\author{
$\checkmark \quad$ Authors \\ vii Conference Committee
}

DLW: HIGH SPEED PRINTING: JOINT SESSION WITH CONFERENCES 11271 AND 11292

1127105 Impact of massive parallelization on two-photon absorption micro- and nanofabrication [1 12 27 $1-4]$

3D MICRO-NANO PRINTING II: FORWARD TRANSFER

$112710 \mathrm{C} \quad$ High throughput LIFT printing of electric circuitry (Invited Paper) [1 1271-1 1$]$

POWDER-BED SLM METAL PRINTING I

$11271 \mathrm{OH} \quad$ Multi-laser fusion process with pre-heating for additive manufacturing [1 1271-17]

$1127101 \quad$ Process development for laser powder bed fusion of pure copper [1 $1271-46]$

POWDER-BED SLM METAL PRINTING II

$112710 \mathrm{~J} \quad$ Additive manufacturing with green disk lasers (Invited Paper) [11271-18]

$11271 \mathrm{OL} \quad$ Measurement of mass loss, absorbed energy, and time-resolved reflected power for laser powder bed fusion (Invited Paper) [1 $1271-20]$

MULTI-MATERIAL PRINTING AND LASER CLADDING

$1127100 \quad$ OCT sensor for layer height control in DED using SINUMERIK® controller (Invited Paper) [1 127 1 23 -23]

11271 OP Substrate influence in laser blown powder of nickel superalloys [ $111271-24]$ 


\section{GLASS 3D PRINTING}

11271 OS Application of glass 3D printing using direct laser melting for fabrication of photonic sensors (Invited Paper) [1 1271-27]

11271 OT Laser powder bed fusion of glass: a comparative study between $\mathrm{CO}_{2}$ lasers and ultrashort laser pulses [1 1271-28]

11271 OU Optical fibers fabricated from 3D printed silica preforms [1 1271-29]

$112710 \mathrm{~V}$ Laser-based drawing method for posture-free objects by photochromic active marking with high-speed coaxial gaze control [1 1271-30]

\section{NOVEL DEVICES AND BIOSENSOR PRINTING}

$112710 X \quad$ Manufacturing of molds by multiphoton polymerization for micro-replication of optically enhanced surfaces [1 $11271-34]$

\section{BIOSTRUCTURE 3D PRINTING}

$1127111 \quad$ Fabrication of 3D fibre scaffolds for tumor-immune system interactions by two photon polymerisation [1 1 $1271-38]$

\section{POSTER SESSION}

1127114 Development of blue diode laser for additive manufacturing [1 1271-41]

1127116 Fabrication of tunable and wearable strain sensor for adjusting photo-polymerization [1 1227 1-43]

1127117 Forming of pure copper rod by LMD method with blue diode lasers [1 1271-44] 


\section{Authors}

Numbers in the index correspond to the last two digits of the seven-digit citation identifier (CID) article numbering system used in Proceedings of SPIE. The first five digits reflect the volume number. Base 36 numbering is employed for the last two digits and indicates the order of articles within the volume. Numbers start with $00,01,02,03,04,05,06,07,08,09,0 A, 0 B \ldots . .0 Z$, followed by 10-12, 20-2Z, etc.

Abe, N., 14, 17
Ares, Félix, OX
Arnoux, Caroline, 05
Baldeck, Patrice, 05
Camacho Rosales, Angeles L., OU
Carlier, Quentin, 05
Chen, Yizheng, OS
Chorvat, D., 11
Cohen, Sharona Sedghani, OC
Coto, Ivette, OX
Coupek, Daniel, OJ
Cunderlikova, B., 11
Deisenroth, David C., OL
Delgado, Tamara, OX
Donovan, Simon, OP
Ermak, Oleg, OC
Franke, Joerg, OI
Funada, Y., 14, 17
Gontad, Francisco, OX
Hara, T., 17
Hayakawa, Tomohiko, OV
Heggarty, Kevin, O5
Heidler, Nils, OT
Higashino, R., 14, 17
Hilbert, Fabian, O5
Hilpert, Enrico, OT
Houbertz, Ruth, 05
Hua, Liwei, OS
Hwang, S., 16
Hwang, T., 16
Ishikawa, Masatoshi, OV
Kogel-Hollacher, Markus, 0O
Kong, H., 16
Kosnac, D., 11
Kotler, Zvi, 0C
Lane, Brandon, OL
Lei, Jincheng, OS
Mantei, Willi, 05
Masuno, S., 14
Mateasik, A., 11
Matthäus, Gabor, OT
McAuliffe, Bryan, OP
Mekhontsev, Sergey, OL
Mikawa, Yuri, OV
Monnereau, Cyrille, 05
Mouchard, Adrien, OP
Myrell, Anne, OJ
Nolte, Stefan, OT
Núñez Velázquez, Martin M. A., OU

Ono, K., 17

Otero, Nerea, $0 \mathrm{X}$

Peled, Itay, 0C

Perez Covarrubias, Luis, 05

Pomeroy, Michael J., OP

Regulin, Daniel, 00

Risse, Jeroen, $\mathrm{OH}$

Robinson, Jeremy, OP

Romero, Pablo, OX

Sahu, Jayanta K., OU

Sato, Y., 14, 17

Schade, Lisa, OT

Scherbaum, Tobias, oJ

Schmiedeke, Samuel, ol

Schneider, Heinz-Ingo, 00

Schweikert, Sven, $\mathrm{OH}$

Seyfarth, Brian, OT

Shobu, T., 14

Staudenmaier, Christian, 00

Stender, Benedikt, 05

Stoll, Thomas, Ol

Strebel, Matthias, 00

Sueishi, Tomohiro, OV

Tang, Jianan, OS

Tanner, David, OP

Teplicky, T., 11

Thielmann, Michael, OJ

Trautnitz, Pascal, Ol

Travitzky, Nahum, ol

Tsukamoto, M., 14, 17

Ullsperger, Tobias, OT

Vidal, Sara, OX

Wagenblast, Philipp, $\mathrm{OH}, \mathrm{OJ}$

Wiedenmann, Jonas, 05

Wu, Yongji, OS

Xiao, Hai, OS

Yeo, J., 16

Zaiss, Jörg, $\mathrm{OH}$

Zhang, Qi, OS

Zhao, Xiao, OU

Núñez Velázquez, Martin M. A., OU 
Proc. of SPIE Vol. 11271 1127101-6

\section{Downloaded From: https://www.spiedigitallibrary.org/conference-proceedings-of-spie on 26 Apr 2023
Terms of Use: https://www.spiedigitallibrary.org/terms-of-use}




\section{Conference Committee}

Symposium Chairs

Beat Neuenschwander, Berner Fachhochschule Technik und Informatik (Switzerland)

Xianfan Xu, Purdue University (United States)

Symposium Co-chairs

Craig B. Arnold, Princeton University (United States)

Takunori Taira, Institute for Molecular Science (Japan)

Program Track Chairs

Bo Gu, Bos Photonics (United States)

Stefan Kaierle, Laser Zentrum Hannover e.V. (Germany)

Conference Chairs

Bo Gu, Bos Photonics (United States)

Hongqiang Chen, GE Global Research (United States)

Conference Co-chair

Henry Helvajian, The Aerospace Corporation (United States)

Conference Program Committee

Corey M. Dunsky, Aeos Consulting, Inc. (United States)

John T. Fourkas, University of Maryland, College Park (United States)

Youping Gao, Aerojet Rocketdyne (United States)

Andreas Heinrich, Hochschule Aalen (Germany)

Weidong Huang, Northwestern Polytechnical University (China)

Linas Jonušauskas, Femtika UAB (Lithuania)

Edward C. Kinzel, Missouri University of Science and Technology (United States)

Christoph Leyens, Fraunhofer-Institut für Werkstoff- und Strahltechnik IWS (Germany)

Jian Liu, PolarOnyx, Inc. (United States)

Shuang Liu, Miller Electric Manufacturing Company (United States)

Henry Peng, Soochow University (China)

Alberto Piqué, U.S. Naval Research Laboratory (United States)

Yuji Sano, Institute for Molecular Science (Japan)

Michael Thiel, Nanoscribe GmbH (Germany) 
Andrea Toulouse, Institut für Technische Optik (Germany)

Paul S. Unwin, Stanmore Implants (United Kingdom)

Augustine M. Urbas, Air Force Research Laboratory (United States)

Martin Wegener, Karlsruher Institut für Technologie (Germany)

\section{Session Chairs}

1 DLW: Microoptics and Metals: Joint Session with Conferences 11271 and 11292

Georg von Freymann, Technische Universität Kaiserslautern (Germany)

2 DLW: High Speed Printing: Joint Session with Conferences 11271 and 11292

Harald Giessen, Universität Stuttgart (Germany)

3 3D Micro-Nano Printing I: Multi-Photon Polymerization

Hongqiang Chen, GE Global Research (United States)

$4 \quad 3 D$ Micro-Nano Printing II: Forward Transfer

Kristin M. Charipar, U.S. Naval Research Laboratory (United States)

5 Powder-Bed SLM Metal Printing I

Hongqiang Chen, GE Global Research (United States)

6 Powder-Bed SLM Metal Printing II

Henry Helvajian, The Aerospace Corporation (United States)

7 Multi-Material Printing and Laser Cladding

Henry Helvajian, The Aerospace Corporation (United States)

8 Glass 3D Printing

Linas Jonušauskas, Femtika UAB (Lithuania)

9 Novel Devices and Biosensor Printing

Andrea Toulouse, Institut für Technische Optik (Germany)

10 Biostructure 3D Printing

Martin Wegener, Karlsruher Institut für Technologie (Germany) 